\title{
„Für Frauen gibt es immer Arbeit!“ - Eine intersektionale Analyse migrantischer Arbeit in Spanien
}

\author{
Johanna Neuhauser
}

(C) Der/die Autor(en) 2020

Zusammenfassung Eine der häufigsten Antworten, die ich bei meiner Feldforschung in Spanien auf die Frage bekommen habe, wie sich die ökonomische Krise 2008 auf die Arbeitssituation von Migrantinnen ausgewirkt hat, war: „Für Frauen gibt es immer Arbeit“. Im folgenden Beitrag wird diese generalisierende Aussage einer genaueren Betrachtung unterzogen und die Auswirkungen der Krise werden entlang von Geschlecht und Ethnizität untersucht. Ziel des konzeptionellen Artikels ist, zu zeigen, dass eine intersektionale Analyse migrantischer Arbeit einen privilegierten Zugang zu grundlegenden gesellschaftlichen Widerspruchskonstellationen eröffnet und verdeckte Herrschaftszusammenhänge sichtbar machen kann. Sie trägt zu einer differenzierten Analyse von Arbeitsausbeutung bei und vermittelt zwischen den strukturellen Mechanismen und der subjektiven Wahrnehmung gesellschaftlicher Ungleichheit.

Schlüsselwörter Intersektionalität · Migration · Krise $\cdot$ Gesellschaftliche Arbeitsteilung 


\title{
“There's always work for women!"-An intersectional analysis of migrant labor in Spain
}

\begin{abstract}
One of the most frequent answers I received in my field research in Spain to the question of how the economic crisis of 2008 has affected the work situation of migrant women was: "There is always work for women". In the paper, this generalizing statement will be examined more closely and the effects of the crisis along gender and ethnicity will be investigated. The aim of the conceptual article is to show that an intersectional analysis of migrant labor can provide privileged access to fundamental social contradictions and reveal hidden power relations. Such investigation contributes to a differentiated analysis of labor exploitation and articulates the structural mechanisms with the subjective perception of social inequality.
\end{abstract}

Keywords Intersectionality $\cdot$ Migration $\cdot$ Crisis $\cdot$ Division of labor

\section{Einleitung}

In Spanien hat die ökonomische Krise von 2008 zu Massenarbeitslosigkeit sowie zu einer Neuausrichtung von einer expansiven hin zu einer restriktiven Migrationspolitik geführt. Nach einem Jahrzehnt, in dem Spanien die höchste Einwanderungsquote in der Europäischen Union verzeichnet hatte, brach mit der Krise die Immigration ein (Reher et al. 2011). Der Kurswechsel in der Migrationspolitik wurde bereits unter der sozialistischen Regierung eingeläutet und mit dem Wechsel zur konservativen Regierung verstärkt. Reguläre Zuwanderungsmöglichkeiten wie das Kontingent zur Anwerbung ausländischer Arbeitskräfte wurden stark beschränkt und die Rückkehr von Migrant_innen forciert (Baumer 2017, S. $211 \mathrm{ff}$.). In dem stark segmentierten Arbeitsmarkt Spaniens wirkten sich die Rezession und die im Zuge der Austeritätsmaßnahmen implementierten Arbeitsmarktreformen vor allem auf jene Sektoren negativ aus, in denen kleinere Unternehmensstrukturen, Saisonarbeit und temporäre Beschäftigung vorherrschen, die Gewerkschaftsbeteiligung niedrig ist und informelle Arbeitsverhältnisse verbreitet sind (Banyuls und Recio 2017). Da Migrierte aus Nicht-EU-Ländern in erster Linie in diese Segmente des Arbeitsmarktes integriert waren, traf sie die krisenbedingte Arbeitslosigkeit besonders stark (Orrenius und Zavodny 2009). Quantitative Untersuchungen des spanischen Arbeitsmarkts weisen zudem darauf hin, dass aufgrund ihrer sektoralen Einordnung in der ersten Phase der Krise männliche Arbeitsmigrant_innen stärker als weibliche von Arbeitslosigkeit betroffen waren (Muñoz 2012; Gil-Alonso und Vidal-Coso 2015).

Wie ich im Folgenden zeigen werde, spiegeln sich diese unterschiedlichen Kriseneffekte entlang von Ethnizität und Geschlecht auch in den Erzählungen der von mir interviewten Migrant_innen aus Lateinamerika wider. Die Entscheidung, die Fallauswahl auf Lateinamerikaner_innen zu beschränken, liegt im historischen Kontext der lateinamerikanischen Arbeitsmigration nach Spanien begründet. Die Befragten sind in der ökonomischen Boomphase Ende der 1990er- und Anfang der 2000erJahre nach Spanien migriert. $\mathrm{Zu}$ dieser Zeit erzeugte das rasche Wachstum in arbeitsintensiven Sektoren wie in der Bauindustrie, im Tourismus und in den perso- 
nenbezogenen Dienstleistungen einen hohen Bedarf an (migrantischer) Arbeitskraft (Cachón und Aysa-Lastra 2015). Im Zuge der gestiegenen Erwerbsbeteiligung von spanischen Frauen entstand eine große Nachfrage nach bezahlter Haushaltsarbeit. Aus diesem Grund waren Frauen die Pionierinnen der lateinamerikanischen Migration nach Spanien, wobei in der folgenden Phase ein Gleichgewicht zwischen den Geschlechtern hergestellt wurde (Oso und Catarino 2013). Politisch wurde die lateinamerikanische Einwanderung durch staatliche Maßnahmen wie Regularisierungsprogramme mit besonders günstigen Resolutionen für Einwanderer_innen aus Lateinamerika gefördert (Araujo und Pedone 2013). Vor diesem historischen Hintergrund sind die Erzählungen lateinamerikanischer Migrant_innen, die verschiedene Phasen der spanischen Arbeitsmarkt- und Migrationspolitik erlebt haben und deren Migrationsströme durch eine ausgeprägte Geschlechterdynamik gekennzeichnet sind, besonders geeignet, um die Zusammenhänge zwischen subjektiven Wahrnehmungen, politisch-ökonomischen Entwicklungen und geschlechtsspezifischen Unterschieden zu erfassen.

Die folgende Analyse baut auf Gruppen- und Einzelinterviews auf, die ich in Madrid in den Jahren 2014 und 2017 geführt habe. Insgesamt waren dies 20 semistrukturierte Gruppen- und Einzelinterviews mit lateinamerikanischen Arbeitsmigrant_innen sowie 10 Expert_inneninterviews mit Nichtregierungsorganisationen, Migrant_innenselbstorganisationen und Gewerkschaften. Für diesen Artikel greife ich vor allem auf die Interviews mit lateinamerikanischen Arbeitsmigrant_innen zurück. Diese habe ich im Rahmen ethnographischer Beobachtungen bei lateinamerikanischen Fußballturnieren und in von Lateinamerikaner_innen betriebenen Bars und Restaurants in der Peripherie von Madrid gewonnen. Da die Männer und Frauen oft in kleinen Gruppen zusammenstanden oder -saßen, gelang es, den Großteil der Gruppeninterviews relativ spontan und in einer informellen Atmosphäre durchzuführen. Aufgrund der interaktiven Dynamik, die innerhalb (mehr oder weniger) homogener Kollektive erzeugt wird, trägt diese Forschungsmethode dazu bei, die Rolle der Forschenden bei der Datenerhebung zu dezentralisieren (Kamberelis und Dimitriadis 2013, S. 15f.). Da es mir darum ging, mittels einer komparativen Analyse kollektive Orientierungsmuster induktiv herauszuarbeiten, habe ich das empirische Material mithilfe der dokumentarischen Methode ausgewertet (Bohnsack 2010). Die Interviewten waren zum Zeitpunkt der Erhebung in drei verschiedenen Arbeitssektoren tätig oder ehemals tätig, in denen migrantische Arbeiter_innen im urbanen Kontext vorrangig beschäftigt sind. Diese Sektoren sind in besonderem Ausmaß von prekären Arbeitsbedingungen geprägt und stark geschlechtlich segmentiert: der Bausektor als von Männern dominierter Sektor, die bezahlte Hausarbeit als feminisiertes Arbeitsfeld und die Hotel- und Gastronomie als gemischt-geschlechtlicher Sektor. In diesem Artikel fokussiere ich aufgrund ihrer kontrastierenden Geschlechterzusammensetzung und Krisenbetroffenheit auf die ersten beiden Sektoren. Ich begegne damit einer Forschungslücke, da komparative Analysen im Bereich migrantischer Arbeit rar sind und Geschlechterperspektiven vor allem auf das Feld der bezahlten Haushaltsarbeit fokussieren, wobei männlich dominierte Sektoren wenig in den Blick genommen werden (Kofman 2013). Gerade in dem geschlechtlich und ethnisch stark segmentierten Arbeitsmarkt Spaniens ist ein vergleichender Blick auf feminisierte und maskulinisierte Arbeitsfelder aufschlussreich, um Persistenzen so- 
wie Verschiebungen sozialer Ungleichheitsverhältnisse sichtbar zu machen. Wie ich zeigen werde, handelt es sich außerdem um Sektoren, die auf unterschiedliche Weise von den Auswirkungen der ökonomischen Krise und den Austeritätspolitiken betroffen waren. Der Bausektor war im Zug des Platzens der Immobilienblase jener Sektor, in dem von 2007 bis 2011 der Verlust an Arbeitsplätzen am massivsten war (Serrano 2012). Dieser Einbruch erfolgte nach einer expansiven Phase (1995-2007) des Beschäftigungswachstums, das zu einem hohen Ausmaß von migrierten Arbeiter_innen aus lateinamerikanischen Ländern, Marokko und Rumänien getragen wurde (Meardi et al. 2012, S. 6). ${ }^{1}$ Im Gegensatz zum stark zyklischen Charakter des Baugewerbes kam es im Sektor der bezahlten Haushaltsarbeit aufgrund des anhaltenden Bedarfs spanischer Familien nach haushaltsnahen Dienstleistungen in der ersten Phase der Krise zu keiner Reduktion von Arbeitsplätzen (Díaz Gorfinkiel 2016). Hinzu kam, dass infolge von politischen Mobilisierungen der Bewegung der Haushaltsarbeiterinnen im Rahmen der ILO-Konvention 189 ein Gesetz für eine umfassende Reform des Sozialversicherungssystems (Ley 27/2011) erlassen wurde, durch das Haushaltsarbeit anderen Arbeitsbereichen weitgehend gleichgestellt wurde (ebd.; Pflücke 2018). Dies führte zwar anfänglich zu Verbesserungen der Arbeitsbedingungen insbesondere dem Anstieg der formal, daher sozialversichert Beschäftigten -, die gesetzliche Änderung wurde in den Folgejahren jedoch zum Teil zurückgenommen (Díaz Gorfinkiel 2016), was zusammen mit den krisenbedingten Veränderungen wie dem Anstieg der Teilzeitarbeit und Lohnkürzungen (UGT 2016) insbesondere in einer zweiten Phase der Krise die Prekarisierung der Arbeitsbedingungen zur Folge hatte.

Bevor ich die empirischen Befunde meine Forschung darstelle, werde ich eine intersektionale Perspektivierung auf Arbeit entwickeln, die es mir erlaubt, gesellschaftstheoretische und polit-ökonomische Ansätze mit der Analyse der subjektiven Sichtweisen der interviewten Arbeitsmigrant_innen zu verbinden. Dabei thematisiere ich zuerst die Fallstricke intersektionaler Ansätze und mache den Begriff der ,intersektionalen Unsichtbarkeit“" als Heuristik für meine empirische Analyse produktiv. Ich beschäftige mich danach mit der Bedeutung, die in den Interviews den Ungleichheitsachsen Ethnizität und Geschlecht für die gesellschaftliche Arbeitsteilung in der Krise zugeschrieben wird, und verbinde diese mit segmentationstheoretischen Perspektiven. Da es insbesondere einer feministischen Analyse von Krisenauswirkungen darum gehen muss, den Fokus sowohl auf die Effekte auf dem Arbeitsmarkt als auch auf unbezahlte Reproduktionsarbeit zu richten, geht mein Blick dabei aber immer auch über einen erwerbsarbeitszentrierten Ansatz hinaus. Übergeordnetes Ziel der Interviewanalyse ist es, danach zu fragen, welche Mechanismen der sozialen Differenzierung und Hierarchisierung qua Ethnizität und Geschlecht zur ungleichen Arbeitsteilung im (Post-)Krisen-Spanien beitragen. Abschließend führe ich die Ergebnisse zusammen und verdeutliche, dass die entwickelte intersektionale Herangehensweise zu einer differenzierten Analyse von Mechanismen der Arbeitsausbeutung beiträgt. Mein Beitrag ordnet sich damit in eine Reihe neuerer Arbeiten ein, die argumentieren, dass arbeitssoziologische Studien, in denen Intersektionalität

\footnotetext{
${ }^{1}$ Schätzungen zufolge waren vor Einbruch der Krise 2008 zirka 30\% der Arbeitskraft in dem Sektor ausländischer Nationalität (Meardi et al. 2012, S. 6).
} 
bisher unzulänglich Eingang gefunden hat, von einem intersektionalen Ansatz im Forschungsdesign und der Datenanalyse profitieren (zum Überblick siehe McBride et al. 2015).

\section{Intersektionaler Analyserahmen für die Untersuchung migrantischer Arbeit in der Krise}

Trotz aller Unterschiede stimmen verschiedene Ansätze von Intersektionalität darin überein, den Blick auf die Mehrdimensionalität und Verwobenheit sozialer Kategorien bzw. Ungleichheitsachsen zu richten. ${ }^{2}$ Eine große Schwierigkeit intersektionaler Ansätze besteht jedoch darin, dass sich diese häufig auf sehr unterschiedlichen Analyse- und damit Abstraktionsebenen bewegen (Anthias 2012, S. 127). Kritisiert wird insbesondere, dass sich die meisten Studien auf die Mikro- bis Mesoebene und damit auf Identitätskonstruktionen oder Fragen von Diskriminierung konzentrieren, während die strukturellen Dimensionen von Intersektionalität kaum ausgeleuchtet werden (Knapp 2012, S. 432f.; McKinzie und Richards 2019, S. 4). Gesellschaftstheoretische Intersektionalitätsansätze sind sich hingegen einig, dass es einer ,Vorstellung vom gesellschaftlichen Gesamtzusammenhang“ (Klinger 2003, S. 249) bedarf, um die Spezifika als auch die Verschränkung gesellschaftlicher Ungleichheitsachsen zu bestimmen. Ich folge diesem Ansatz, plädiere aber zugleich für einen Zugang, der die empirische Untersuchung von alltäglichen Erfahrungen nicht außen vor lässt, sondern die Anschlussstellen zwischen subjektiven und objektiven Dimensionen gesellschaftlicher Ungleichheit ins Zentrum rückt. Mit der Frage nach den unterschiedlichen Analyseebenen von Intersektionalität verbunden ist jene nach der zu bestimmenden Anzahl von Ungleichheitsachsen. Dabei läuft insbesondere eine identitätsbezogene Perspektive Gefahr, potenziell infinite interferierende Kategorien einzubeziehen, was letztlich in einer Untersuchung individueller Differenzen mündet (Anthias 2012, S. 128). Um dies zu verhindern, scheint mir der Ansatz, die gesellschaftliche Arbeitsteilung als Dreh- und Angelpunkt heranzuziehen, zielführend, da über diese Grundmuster gesellschaftlich relevante Ungleichheiten erzeugt werden. Nach Klinger (2003) stimmen unter Bezug auf Arbeit die drei Achsen Klasse, Geschlecht und Race/Ethnizität/Citizenship ${ }^{3}$ mehr als andere Kategorien darin überein, dass über sie „,ein Ungleichheit begründender und legiti-

\footnotetext{
2 Im Folgenden beziehe ich mich vor allem auf die im Rahmen akademischer Diskurse entworfenen Kritiken am Intersektionalitätskonzept. Erwähnt werden muss jedoch auch die bedeutende politische Kritik, dass Intersektionalität als eine ursprünglich von feminists of colour entworfene politische Strategie zwar einen festen Platz in der akademischen Welt gefunden hat, in dieser aber ihre politischen Ursprünge oftmals vergessen werden (z. B. Collins und Bilge 2016).

3 Ich betrachte Race, Ethnizität und Citizenship als Teil einer Ungleichheitsachse, die historisch auf die Kolonialisierung zurückgeht und bis heute fortdauernde koloniale Machtasymmetrien und Ordnungsmuster beschreibt (Quijano 2016). Allerdings akzentuieren die Begriffe jeweils Unterschiedliches: Während Citizenship stärker auf differenzierte In- und Exklusionsmechanismen durch (nationalstaatliche) Grenzen beruht, durch die Menschen in eine Vielzahl rechtlicher Kategorien klassifiziert werden (De Genova 2013), begründen Race und Ethnizität Hierarchisierungen, die nicht an Staatbürgerschafts- oder Aufenthaltsrechte geknüpft sind, denn wie Anderson (2019) bemerkt: „once migration is no longer at the border it becomes ,race“, and minority ethnic citizens are often already ,migrantized““ (ebd., S. 8).
} 
mierender Fremdheitseffekt, d.h. eine Ausgrenzung (Externalisierung) erzeugt wird, mit dem Ziel oder mindestens mit dem Resultat, eine Reduzierung des für die geleistete Arbeit zu entrichtenden Preises herbeizuführen d.h. Ausbeutung stattfinden zu lassen“ (ebd., S. 26). Über die Prekarisierung der Beschäftigungsverhältnisse, die Funktionalisierung von (unbezahlter) Reproduktionsarbeit oder die Differenzierung von Arbeitsrechten entlang des Migrationsstatus lässt sich sicherstellen, dass kontinuierlich kostengünstige Arbeitskraft für besonders prekäre, sozial abgewertete und niedrig entlohnte Arbeiten mobilisiert werden kann (Dörre et al. 2009, S. 562 f.).

Im Kontext der geschlechts- und ethnizitätsspezifischen Auswirkungen der Krise auf Erwerbs- und Reproduktionsarbeit erscheint mir insbesondere die Heuristik einer ,intersectional invisibility“, wie von Gudrun-Axeli Knapp (2013) formuliert, gewinnbringend. Anknüpfend an Marx geht es Knapp dabei darum, die Mechanismen aufzuzeigen, die ein „Unsichtbarwerden und Unsichtbarmachen sozialer Zusammenhänge von Macht, Herrschaft und Ungleichheit" (ebd., S. 252) erzeugen. Dabei reichen die Formen, in denen sich diese Unsichtbarmachung vollzieht, von Naturalisierung, Normalisierung bis hin zur Reifizierung des Gesellschaftlichen. In allen Fällen geht es darum, ein unmittelbar Gegebenes herzustellen, das den gesellschaftlichen Charakter der Zusammenhänge verdeckt (ebd., S. 252). Während nach Marx vor allem die Verdeckung der ungleichen Tauschbedingungen im Verhältnis von Arbeit und Kapital im Zentrum steht, muss aus einer feministischen Perspektive auch die widersprüchliche Verknüpfung im Geschlechterverhältnis - als vor allem durch die gleichzeitige Trennung und wechselseitige Bezogenheit von öffentlich und privat bzw. von Produktion und Reproduktion bedingt - berücksichtigt werden (Becker-Schmidt 2004). In modernen Gesellschaften sind demnach bezahlte und unbezahlte Arbeit in einer Weise verknüpft, ,,dass soziale Unstimmigkeiten im Modus des Zusammenschlusses unsichtbar werden und die damit verbundenen sozialen Zumutungen verborgen bleiben“ (Becker-Schmidt 2009; zitiert in Knapp 2013, S. 237). ${ }^{4}$ Ein weiterer häufig verdeckter Herrschaftszusammenhang ist jener zwischen Globalem Norden und Süden bzw. zwischen Zentren und Peripherien. Aus dekolonialer Perspektive kann die globale Arbeitsteilung nur vor dem Hintergrund des Kolonialismus und seinen sich bis in die Gegenwart fortschreibenden Strukturen verstanden werden (Quijano 2016). Während der globale Kapitalismus arbeitsintensive Produktion auslagert, um sich die Ausbeutung von Arbeitskraft in der Peripherie zunutze zu machen, erfüllen Migrationspolitiken nicht zuletzt die Funktion einer ,globalen Arbeitsmarktpolitik“ (Gutiérrez Rodríguez 2005, S. 74). Der prekäre Status von Migrierten wird den Anforderungen des neoliberalen Kapitalismus dabei besonders gut gerecht, da er als disziplinierendes Instrument dient, um Migrierte zu besonders flexiblen Arbeiter_innen zu machen (Mezzadra 2011). In diesem Sinn muss die Stratifizierung von Rechten durch die Migrations- und Grenzregime als zentraler Mechanismus verstanden werden, der die Arbeit von Migrant_innen zu ei-

\footnotetext{
${ }^{4}$ Da sie im ungleich höheren Ausmaß die Arbeit in Haushalt und Familie verrichten, treffen diese Zumutungen gesellschaftlich Frauen stärker als Männer, wobei sich ihre ,doppelte Vergesellschaftung“ (BeckerSchmidt 2004) in Erwerbs- und Reproduktionsarbeit in Gefühlen von Widersprüchlichkeit und Ambivalenz ausdrückt.
} 
nem konstitutiven Moment der internationalen Arbeitsteilung werden lässt (Buckel 2012, S. 87).

Neben der Bestimmung der die gesellschaftliche Arbeitsteilung strukturierenden Ungleichheitsachsen besteht eine weitere Herausforderung darin, einerseits die historischen und strukturellen Spezifika dieser Herrschaftsverhältnisse zu berücksichtigen, und diese andererseits analytisch zu verknüpfen. In der gesellschaftlichen Arbeitsteilung folgen insbesondere Geschlecht und Ethnizität im Vergleich zu Klasse anderen Strukturlogiken. Anna Pollert (1996) sieht den grundlegenden Unterschied in der internen Dynamik der Selbstexpansion des Kapitals - des Profits -, die dem Klassenverhältnis unterliegt (ebd., S. 643). Eine solche systemimmanente, sich selbst erhaltende materielle Dynamik könne im Geschlechterverhältnis nicht ausgemacht werden. Denn auch wenn Produktions- notwendigerweise durch Reproduktionsverhältnisse erweitert werden müssen, können Geschlechterverhältnisse nicht auf die gleiche Weise abstrakt aus Zweiteren gefolgert werden, da - wie Pollert pointiert bemerkt - Kapitalist_innen nicht einfach aufhören könnten, Lohnarbeit auszubeuten. Männer hingegen könnten durchaus die geschlechterhierarchische Arbeitsteilung herausfordern und damit zu einer Neugestaltung des Zusammenhangs von Produktion und Reproduktion beitragen (ebd.). Daraus folgt, dass sich Geschlecht und Ethnizität insofern ähneln, als bei beiden Unterschiede konstruiert werden müssen, die ihrerseits gesellschaftliche Ungleichheit erst herstellen oder legitimieren (Ross 2008, S. 36). Um zu erklären, wie es dazu kommt, dass verschiedene Arbeitssektoren als männlich oder weiblich bzw. als migrantisch oder nicht-migrantisch typisiert werden und warum Frauen häufiger als Männer und Migrant_innen häufiger als Nicht-Migrant_innen abgewertete Arbeiten ausführen, braucht es daher die Verschränkung von gesellschaftstheoretischen Analysen mit sozialkonstruktivistischen Ansätzen. In diesem Sinn hat die arbeitssoziologische Geschlechterforschung darauf hingewiesen, dass ein Wechselverhältnis zwischen der Organisation von Arbeit und der Konstruktion von Geschlecht besteht (Teubner 2010). Angelika Wetterer (2002) zufolge stellt die geschlechtliche Arbeitsteilung sogar den ,,zentrale[n] Modus der sozialen Konstruktion von Geschlecht“ (ebd., S. 26) dar. Denn indem Frauen und Männer Unterschiedliches tun, werden sie auch zu Verschiedenen (ebd., S. 523).

Bis auf den Widerspruch von Arbeit und Kapital - also der Ungleichheitsachse Klasse - werden gesellschaftliche Spaltungen entlang von Geschlecht und Ethnizität daher nicht durch den Kapitalismus erzeugt, sondern kapitalistische Herrschaftslogiken bedienen sich der geschlechtlichen und ethnisierten Segmentierung des Arbeitsmarkts und der Abwertung feminisierter und rassifizierter Arbeit und verstärken diese (Peck 1996, S. 65). Einem marxistischen Verständnis folgend beziehe ich den Begriff der Klasse zwar primär auf eine polit-ökonomische Relationalität, die wesentlich durch die Verfügungsmacht über Arbeit und die Positionierung im Erwerbssystem bestimmt ist (Knapp 2012, S. 438), plädiere jedoch zugleich für eine analytische Erweiterung des Begriffs, durch die auch kulturelle oder soziale Ressourcen und Distinktionsformen berücksichtigt werden. Wenngleich Lohnarbeit nach einer kapitalistischen Strukturlogik angeeignet wird, folgen die maßgebenden Prozesse, wie diese Aneignung vonstatten geht, keinem Automatismus und können daher nicht a priori festgelegt werden, sondern sie müssen als soziale Praktiken 
kontextsensibel untersucht werden. ${ }^{5}$ Diese kontextspezifischen Prozesse verlaufen innerhalb bestehender Geschlechter- und Nord-Süd-Verhältnisse und werden durch diese daher wesentlich mitkonstituiert. Wie jedoch diese Verknüpfung bzw. Ko-Konstituierung der unterschiedlichen Ungleichheitsachsen empirisch und theoretisch erfasst werden kann, bleibt nach wie vor eine Herausforderung, die in vielen Arbeiten unzureichend reflektiert wird (kritisch dazu: Jorba und Rodó-Zárate 2019). Floya Anthias (2012) bemerkt beispielweise kritisch, dass die Metapher der Intersektion insofern missverständlich ist, als sie suggeriere, es gebe separierbare Achsen, die sich erst in einem zweiten Schritt kreuzten und Ungleichheiten produzierten. Realiter seien die Kategorien aber häufig analytisch nicht voneinander zu trennen, da sie sich immer schon wechselseitig konstituierten (ebd., S. 128). Der Fokus auf die Überkreuzung von Kategorien bzw. Ungleichheitsachsen laufe Gefahr, diese als gegebene vorauszusetzen und sie damit zu essentialisieren, anstatt ihre Formierung selbst zu untersuchen (ebd.). Gleichzeitig tendiere eine Konzeption von Intersektionalität als „Ko-Konstituierung“ sozialer Kategorien aber auch dazu, die Spezifika der einzelnen Ungleichheitsachsen zu verkennen (ebd., S. 129). Es braucht daher eine empirische Herangehensweise, die sowohl sensibel für die Spezifika der unterschiedlichen Ungleichheitsachsen als auch für ihre praktisch häufig untrennbare Verbindung und wechselseitige Konstituierung ist. Wenn ich im Folgenden die Krisenauswirkungen entlang von Ethnizität und Geschlecht gesondert untersuche, dann betrachte ich dies als eine analytische Trennung, die aber immer wieder durchbrochen werden muss. Den großen Vorteil einer intersektionalen Perspektivierung sehe ich darin, die Mehrdimensionalität von Herrschaftsverhältnissen zu erkennen, indem die Aufmerksamkeit auf das jeweils andere, Unterbelichtete gelenkt wird. Das heißt, in meinem konkreten Untersuchungsfeld - wo die Kriseneffekte auf den ersten Blick vor allem qua Klasse ungleich verteilt erscheinen - gilt es, die ethnisierten Unterschiede näher zu beleuchten, die aufgrund der Stratifizierung von Rechten durch die Migrationsregime und die Stereotypisierung ,migrantisierten Arbeitsvermögens“ entstehen. Um gleichzeitig die ethnisierten Unterschiede nicht zu generalisieren, braucht es wiederum eine Perspektive auf die klassen- und geschlechtsspezifischen Ungleichheiten. Es handelt sich daher um eine potenziell unabgeschlossene Analyse, was eine intersektionale Vorgehensweise besonders herausfordernd macht.

Neben der analytischen Verknüpfung der Ungleichheitsachsen besteht eine weitere Herausforderung darin, die Fallhöhe zwischen strukturellen und subjektiven Dimensionen von Intersektionalität zu vermitteln. Denn im subjekttheoretischen Horizont sind ,die ,Achsen der Differenz ' potenziell anders aufeinander bezogen als im Horizont einer gesellschaftstheoretischen Analyse sozialer Strukturzusammenhänge“ (Knapp 2012, S. 210). Um zu untersuchen, wie sich makrosoziologische Ungleichheitsverhältnisse entlang von Geschlecht, Klasse und Ethnizität in die alltäglichen Erfahrungen und das Handeln der Subjekte übersetzen sowie sie auch umgekehrt durch diese reproduziert, aber auch herausgefordert werden, bedarf es einer dynamischen Analyse, in der ein Wechselspiel von deduktiven und induktiven

\footnotetext{
5 Zur Notwendigkeit kontextbezogener Intersektionalitätsanalysen siehe auch die Ausführungen von McKinzie und Richards (2019).
} 
Verfahren vollzogen wird. ${ }^{6}$ Ich schlage daher vor, die Ungleichheitsachsen einerseits als gesellschaftstheoretischen Horizont vorauszusetzen, vor dessen Hintergrund das empirische Material untersucht und eingeordnet wird. Andererseits gilt es, die ungleichheitsrelevanten Kategorien induktiv aus dem empirischen Material herauszuarbeiten und in Zusammenhang mit dem gesellschaftstheoretischen Bezugsrahmen zu bringen. Weil sich im Moment der Krise strukturelle Widersprüche verdichten (Demirovic und Maihofer 2013, S. 31 f.), eignet sich dieser analytisch besonders gut dazu, die Verbindung von objektiven Strukturen und subjektiven Wahrnehmungs- und Handlungsweisen herauszuarbeiten. In Wirtschaftskrisen verstärkt sich die der kapitalistischen Vergesellschaftung inhärente Logik der Externalisierung: Um Krisen zu bearbeiten, ist es typisch, Kosten und Verluste auf andere Gruppen oder Systeme zu verlagern (Neuhauser 2018, S. 459f.). Am Beispiel Spaniens wird besonders deutlich, dass die zur Bearbeitung der Krise getroffenen Flexibilisierungsmaßnahmen zulasten von Arbeiter_innen gehen, insbesondere jenen in prekären Arbeitsmarktsegmenten (Román 2018, S. 333). In Bezug auf den Arbeitsmarkt fand die „Bekämpfung der Krise“ vor allem über Arbeitsmarktreformen in den Jahren 2010 und 2012 statt, die unter anderem eine Lockerung des Kündigungsschutzes und Lohnkürzungen im öffentlichen Sektor vornahmen (Román 2018; Rosnick und Weisbrot 2015). Ich sehe Krise daher als einen Kristallisationspunkt, an dem die strukturellen Mechanismen dieser Auslagerung akzentuiert auf der Subjektebene erfahren und damit auch dechiffriert werden können. Um strukturelle und subjektive Perspektiven auf gesellschaftliche Ungleichheit empirisch miteinander zu verzahnen, erscheint der Fokus auf Widersprüche und Ambivalenzen, die sich aus den kriseninduzierten Veränderungen der Arbeitsverhältnisse für die Betroffenen ergeben, methodisch aufschlussreich. Außerdem verweisen Verallgemeinerungen, Naturalisierungen und die Darstellung von Selbstverständlichkeiten auf Verdeckungszusammenhänge, die den Subjekten als Erklärungen und Rechtfertigungen für Ungleichheitslagen dienen.

\section{Empirische Analyse von Ungleichheitsachsen am Beispiel migrantischer Arbeit in Spanien}

Wie ich in der folgenden Analyse zeigen werde, spiegeln sich die in der wissenschaftlichen Literatur diskutierten und oben skizzierten Kriseneffekte entlang von Ethnizität und Geschlecht auch in den Erzählungen der befragten Migrant_innen wider. Allerdings wird in den Interviews diese Differenzierung häufig erst in einem zweiten Schritt vorgenommen. So besteht eine große Gemeinsamkeit in den Erzählungen der in den beiden Sektoren beschäftigten Migrant_innen darin, Krise als universelles Phänomen darzustellen, das alle Arbeitnehmer_innen auf dem spanischen Arbeitsmarkt betreffe. Indem sie die Prekarität aller - sowohl der Migrierten als auch der in Spanien geborenen Angehörigen der Arbeiterklasse - hervorheben, betonen die Interviewten das Klassenargument stärker als die ethnisierte und ge-

\footnotetext{
${ }^{6}$ Das entspricht dem Zugang, den Degele und Winker (2011) als das gleichzeitige Offenhalten der Kategorien auf der Identitäts- und Repräsentationsebene und das Festlegen der gesellschaftlich relevanten Herrschaftsverhältnisse auf der Strukturebene bezeichnet haben (ebd., S. 87).
} 
schlechtliche Arbeitsteilung. Dies reflektiert nicht zuletzt quantitative Befunde, die zeigen, dass von 2007 bis 2015 der GINI-Index als Maß für gesellschaftliche Ungleichheit um 15,6\% gestiegen ist (Oxfam 2017, S. 11). Spanien ist damit nach Zypern das Land in der Europäischen Union, in dem die ökonomische Ungleichheit am stärksten gewachsen ist (ebd.). Vor dem Hintergrund dieser zunehmenden Polarisierung ziehen die Interviewten eine besonders scharfe Linie zwischen Arbeitnehmer_innen und Arbeitgeber_innen. Sie kritisieren, dass ihre Arbeitgeber_innen die Krise dazu nutzen, Lohnkürzungen zu legitimieren und die Ausbeutung ihrer Arbeitnehmer_innen zu steigern (Neuhauser 2018, S. 457). Erst im Laufe der Interviews wird die konstatierte Universalität der Krise insofern relativiert, als häufig eingewendet wird, ihre Effekte träfen verschiedene soziale Gruppen in ungleichem Ausmaß. Ebenso sei die Fähigkeit, sich gegen ökonomische Notlagen zu wappnen, unterschiedlich (ebd., S. 456f.). Krise wird daher auf den zweiten Blick als ein sozial hierarchisiertes Phänomen differenziert, das sowohl in Bezug auf Ethnizität als auch Geschlecht unterschiedliche Betroffenheiten hervorruft. Auf einer verdichteten Analyse des empirischen Materials basierend, diskutiere ich im Folgenden exemplarisch Ausschnitte aus den Interviews, welche die intersektionalen Mechanismen der Arbeitsausbeutung besonders gut zum Ausdruck bringen.

\section{Die Funktionalität von Citizenship und der Ethnisierung „migrantischen Arbeitsvermögens“،}

In dem folgenden Interviewausschnitt diskutieren die beiden Freundinnen Zoila und Rafaela über die Frage, inwieweit sich die Krise auf die Arbeitsbedingungen von Migrierten und Spanier_innen auf unterschiedliche Weise ausgewirkt habe. Beide Frauen sind Ende der 1990er-Jahre von Ecuador nach Spanien migriert und im Bereich der Reinigung und haushaltsnahen Dienstleistungen tätig.

Zoila: Die Krise hat die Arbeit beeinflusst ...

Rafaela: ... die Arbeit, weil sie [die Arbeitgeber] nur noch Leute von hier einstellten. Die Migranten wollten sie nicht [mehr].

Zoila: Die Sache ist die, wir Immigranten, wir machen alles. Ein Haus renovieren, sich um alte Leute kümmern, egal was. [...] Die Spanier werden nicht machen, was wir tun. Die meisten tun das nicht. Das rettet uns, denn es gibt uns Arbeit. (Rafaela und Zoila 29.01.2017, S. 11 f.)

Zwischen den beiden Frauen besteht keine Einigkeit darüber, inwiefern sich die Krise unterschiedlich auf spanische und migrantische Arbeiter_innen ausgewirkt hat. Zum einen werden Migrierte als besonders von der Krise betroffen dargestellt, da, wie Rafaela konstatiert, Arbeitgeber_innen vermehrt einheimische Arbeiter_innen bevorzugten. Zum anderen wendet Zoila ein, dass viele Migrant_innen deshalb ihre Arbeit behielten, da sie weniger anspruchsvoll seien und - im Gegensatz zu spanischen Arbeiter_innen - jede Arbeit machen würden. Diese zwei diametralen Positionen zur unterschiedlichen Krisenbetroffenheit entlang von Ethnizität finden sich auch in weiteren Interviews. Dieselbe Kontroverse spiegelt sich in theoretischen Debatten zu den unterschiedlichen Auswirkungen der ökonomischen Rezession auf 
einheimische und migrantische Arbeiter_innen wider (Gil-Alonso und Vidal-Coso 2015). Während zum einen der Befund der höheren Arbeitslosigkeit von Migrierten herangezogen wird, um - in Anlehnung an Marx - die Bildung einer neuen ,industriellen Reservearmee" festzustellen (Beets und Willekens 2009; Castles 2011), wurde zum anderen ins Treffen geführt, dass Migrierte insbesondere in einer ersten Phase weniger betroffen waren, da sie sich beispielsweise besser an prekäre Arbeitsmarktbedingungen anpassten (Cachón und Aysa-Lastra 2015, S. 36 ff.). Diese größere Anpassungs- und Leistungsbereitschaft von migrierten im Vergleich zu spanischen Arbeiter_innen ist ein Orientierungsmuster, das in den Interviews wiederkehrend hervortritt. Lateinamerikaner_innen werden beispielweise als „härter“ und körperlich „stärker“ bezeichnet. Dabei überlagern sich häufig Zuschreibungen körperlicher Härte oder Stärke mit psychologischen Charakteristika eines größeren Durchhaltevermögens bzw. einer höheren Akzeptanz gegenüber prekären Arbeitsbedingungen. So ist zum Beispiel Filipe, der sich und seine Familie mit temporären, meist undokumentierten Aufträgen im Bausektor durchbringt, der Ansicht:

Wir Immigranten sind für alles gut [...] Arbeit, Arbeit, Arbeit. [...] Ach, die Spanier wollen ruhiger leben. [...] An einem Feiertag arbeitet ein Spanier nie. Ein Immigrant macht es, weil er essen will. Für uns ist es auch schwieriger, eine Wohnung zu bekommen, sie fragen uns nach mehr Dingen. (Flavio 05.02.2017, S. 4)

Zum einen wird hier die Akzeptanz jeglicher Arbeitsbedingungen (,für alles gut sein") in Kontrast zur Arbeitseinstellung der spanischen Arbeiter_innen gesetzt. Ethnisierte Konstruktionen werden vor allem dahingehend genutzt, Migrierten eine besondere Bereitschaft, prekäre Arbeitsbedingungen zu akzeptieren, zu attestieren. Das entspricht jener Literatur, die sich zur Erklärung von Arbeitssegmentierung weniger mit den polit-ökonomischen Mechanismen der Nachfrageseite, als vielmehr mit dem Zusammenwirken von Angebot und Nachfrage und der Rolle von ethnisierten Einstellungspraktiken beschäftigt (Waldinger und Lichter 2003; Peck 1996). Dabei sind für die Benachteiligung (oder Bevorzugung) am Arbeitsmarkt sozial zugeschriebene Qualifikationen meist entscheidender als tatsächliche (Peck 1996, S. 30). Insbesondere in prekären Jobs hätten Migrierte oft deshalb Vorrang vor lokalen Arbeiter_innen, weil sie ihren Platz in der sozialen Hierarchie am Arbeitsplatz kannten und damit ihre Unterordnung eher akzeptierten (Waldinger und Lichter 2003, S. 163). In der komparativen Analyse der Interviews wird außerdem deutlich, dass das Orientierungsmuster einer übergeordneten Bereitschaft, Arbeitsleistung auch unter widrigen, ausbeuterischen Bedingungen zu erbringen, nicht nur mit der Intention, die eigenen Jobchancen am Arbeitsmarkt zu erhöhen, sondern auch mit den Migrationsbiografien der interviewten Arbeitsmigrant_innen verbunden ist. Arbeit zu haben, stellt für die in Zeiten der wirtschaftlichen Anwerbung nach Spanien migrierten Lateinamerikaner_innen die zentrale Legitimation für ihren Aufenthalt im Emigrationsland her. Diese Haltung steht nicht zuletzt im Zusammenhang mit einer insbesondere in Krisenzeiten virulent werdenden Migrationspolitik, in der Migrierte nicht als Bürger_innen, sondern als Gastarbeiter_innen betrachtet werden, die in ihre Herkunftsländer zurückkehren sollten, wenn sie am Arbeitsmarkt nicht mehr gebraucht werden (Baumer 2017, S. 213). 
Der interviewte Bauarbeiter geht außerdem über bloße ethnisierte Essentialisierungen einer besonderen ,migrantischen“ Arbeitshaltung hinaus, wenn er diese mit einer größeren materiellen Notwendigkeit (,,essen wollen“) und Schwierigkeiten verknüpft, mit denen Migrierte zum Beispiel am spanischen Wohnungsmarkt konfrontiert sind. Andere Interviewte sehen die Gründe, warum migrantische Arbeiter_innen einfacher auszubeuten sind, in fehlenden - vor allem familialen - Netzwerken, was sie insbesondere in ökonomischen Notlagen vulnerabler macht. Diese Vulnerabilität verstärkt sich zudem dadurch, dass sie oft selbst Rücküberweisungen an die Familienmitglieder im Herkunftsland senden. Große Einigkeit zwischen den Interviewten besteht außerdem in dem Punkt, dass es einen großen Unterschied macht, ob man „Papiere“, daher das unbegrenzte Aufenthaltsrecht oder gar die spanische Staatsbürgerschaft, besitzt oder nicht. Denn auch wenn, wie die Interviewausschnitte zeigen, Ungleichbehandlung auch unabhängig von der Frage nach dem Aufenthaltsstatus über Ethnisierungen fortgeschrieben wird, schafft die formale Gleichheit über Citizenship prinzipiell andere Möglichkeiten, sich gegen ausbeuterische Arbeitsverhältnisse zur Wehr zu setzen. Die durch das Ausländergesetz 2009 in Kraft gesetzte Bedingung, dass in Spanien temporäre Aufenthaltsgenehmigungen nur bei Vorliegen eines Arbeitsvertrags erneuert werden (Torres und Gadea 2015), bekräftigt diesen Zusammenhang. In den Krisenjahren hat sich dadurch nicht nur die Zahl illegalisierter Migrant_innen im Land (Baumer 2017, S. 219), sondern auch der Druck erhöht, prekäre Arbeitsbedingungen zu akzeptieren. ${ }^{7}$ Hinzu kommt, dass die konservative Regierung 2012 den Zugang undokumentierter Migrant_innen zur staatlichen Gesundheitsversorgung stark einschränkte (ebd., S. 334). Diese Beispiele verdeutlichen, wie wichtig es in der Analyse der Arbeitsbedingungen von Migrierten ist, einen über Erwerbsarbeit hinausgehenden Blick auf die im hohen Ausmaß ungleichen Reproduktionsbedingungen und institutionellen Beschränkungen qua Ethnizität und Citzenship-Status zu richten. Es braucht einen historisierenden Zugang zu den migrantischen Erfahrungen, da soziale Prozesse der Diskriminierung und Segregation immer in konkrete Institutionen und Politiken des Arbeitsmarkts, der Organisationen und der Arbeitsplatzkulturen eingebettet sind (Hudson et al. 2017).

\section{Kontinuum der Prekarität - Reproduktion und feminisierte Arbeit in der Dauerkrise}

Während die Frage, inwiefern Migrierte stärker von den kriseninduzierten Veränderungen auf dem Arbeitsmarkt - vor allem von Arbeitslosigkeit - betroffen seien als Spanier_innen, eher kontrovers verhandelt wird, sind sich die Interviewten weitgehend darüber einig, dass Frauen weniger von der Krise betroffen seien als Männer. Die beiden folgenden Ausschnitte von Jimena und Zoila, die beide in Haushalten spanischer Familien arbeiten, stehen beispielhaft für diese Einschätzung.

\footnotetext{
7 Wenngleich ich mit Mezzadra (2011, S. 126) davon ausgehe, dass Irregularität als Teil eines Kontinuums verschiedener Subjektpositionen zu verstehen ist, erachte ich es als ebenso wichtig, analytisch zu differenzieren, wann eher ethnische Zugehörigkeit und wann der Aufenthaltsstatus Diskriminierung strukturieren und wie beide zusammenwirken (Tapia und Alberti 2019, S. 320).
} 
Und natürlich ist es für den Mann schwieriger, einen Job zu bekommen. Für die Frau ist es so: Auch wenn sie eine Haushaltsarbeiterin ist - sie hat alternative Auswege. (Jimena, Família Ecuatoriana 03.02.2017, S. 6)

Ich glaube, für die Männer ist es schwerer, da es keine Arbeit für Männer gibt. Für die Frau, sage ich, wir haben immer die Möglichkeit ... wir schauen hier, wir schauen da - es gibt was [Arbeit]! Für den Mann nicht, denn er sucht oft ein Jahr, ohne etwas zu finden. Mein Freund findet seit ungefähr fünf Jahren keinen Job. (Zoila, Rafael und Zoila 29.01.2017, S. 5)

Die uneingeschränkte Verfügbarkeit von Arbeitsplätzen für Frauen wird von den Interviewten erstens mit der Erwerbsmöglichkeit von Frauen in privaten Haushalten und den massiven Jobverlusten von Männern im Bausektor in Verbindung gebracht. Diese subjektive Einschätzung entspricht dem Befund, dass in Europa haushaltsnahe Dienstleistungen weniger von krisenbedingter Arbeitslosigkeit betroffen waren (Farris 2012). Den Grund hierfür sieht Sara Farris in der Besonderheit des Sektors der häuslichen Dienstleistungen, die in der ,nicht austauschbare[n] Materialität der affektiven [...] Arbeit“" liege, die es den Arbeitgeber_innen erschwere, die Arbeitnehmer_innen zu ersetzen, sobald eine Vertrauensbeziehung bestehe (ebd., S. 190). Außerdem haben Faktoren wie der demografische Wandel, der gestiegene Bildungsstand und die wachsende Erwerbsbeteiligung von spanischen Frauen sowie die schwache öffentliche Infrastruktur an Betreuungseinrichtungen in dem Sektor eine Nische für weibliche Arbeitsmigrant_innen eröffnet (Araujo und GonzálezFernández 2014). ${ }^{8}$ Zweitens rufen die Interviewten Eigenschaften von Frauen auf, die das Finden von Erwerbsarbeit selbst in ökonomisch schwierigen Zeiten erleichterten. Für die Interviewten charakterisiert größeres Durchhaltevermögen daher nicht nur Migrierte stärker als einheimische Arbeiter_innen, sondern auch Frauen stärker als Männer. Dies wird vor allem in Selbstbeschreibungen der interviewten Frauen wie „wir sind stärker“, „wir sind flexibler“ oder „wir halten mehr aus“ deutlich. Außerdem werden Frauen als positiv, hoffnungsvoll und an der Familie und dem Gemeinwohl interessiert gezeichnet, was häufig einer starken Abwertung von Männern als negativ, verzweifelt, egoistisch und potenziell gewalttätig gegenübersteht. So differenziert beispielsweise eine Haushaltsarbeiterin wie folgt zwischen den Geschlechtern: „Der Mann ist der Erste, der sagt: Lass uns gehen [ins Herkunftsland]. Denn er ist schwächer, [...] die Frau ist die Einzige, die sich für ihre Familie opfert, für ihre Kinder, für all das“ (Gabriela 15.11.2014, S. 10). Diese binären Essentialisierungen orientieren sich nicht nur an gängigen Geschlechterstereotypen, sie werden auch damit begründet, dass sich die familiale Position vieler migrantischer Frauen dahingehend verändert habe, als sie zu Hauptverdienerinnen in ihren Familien geworden sind. Die Haushaltsarbeiterin Gabriela thematisiert die Familientrennungen und die damit verknüpfte „Feminisierung der Ernährungsverantwortung“ (Wichterich 2010) im Zuge der Krise wie folgt:

\footnotetext{
8 Dass dies kein auf den spanischen Kontext beschränktes Phänomen ist, haben einschlägige Forschungsarbeiten zum Zusammenhang zwischen feminisierten Migrationsbewegungen und der Krise der sozialen Reproduktion in Europa gezeigt. Siehe dazu den Beitrag von Haubner in diesem Heft.
} 
Ehen gingen zugrunde, Trennungen, rechtliche Trennungen und Trennungen, wenn der Mann zurückgeht [...] Die Vereinbarung von Arbeit und Familie existiert für die Hausangestellten nicht. Und klar, jetzt noch weniger. Warum? Ich verdiene weniger, daher muss ich mehrere Jobs suchen, [...] um überleben zu können. [...] Die Kinder sind allein [zuhause]. Ein achtjähriges Kind, das sich um die kleineren Kinder kümmert, weil es keine Möglichkeit gibt, jemanden zu bezahlen, der sich kümmert. (Gabriela 15.11.2014, S. 8)

Das zeigt, dass über die „Krise der Arbeit“ hinaus - im Sinne eines erweiterten feministischen Reproduktionsverständnisses (Bakker und Gill 2003) - die umfassenden Reproduktionsbedingungen der Migrant_innen in den Blick genommen werden müssen. Wie der Interviewausschnitt veranschaulicht, leiden unter der „Krise der Reproduktion“"Migrant_innen und dabei vor allem Frauen als Hauptverantwortliche in diesem Bereich in besonderem Ausmaß. Denn ihr soziales Netzwerk ist weniger ausgeprägt, wodurch sie in der Betreuung von Kindern oder Pflegebedürftigen weniger als die lokale Bevölkerung auf private Unterstützungsleistungen durch Familie oder Freund_innen zurückgreifen können. Dadurch treffen die im Rahmen der Austeritätspolitik veranlassten Kürzungen des öffentlichen Betreuungsangebots migrantische Familien besonders hart.

Neben der Festlegung von geschlechtsspezifischen Eigenschaften, die in den Interviews insbesondere von den befragten Frauen vorgenommen wird, unterscheidet sich die Art und Weise, wie über die Krise, über Arbeitslosigkeit und Arbeitsplatzunsicherheit gesprochen wird, stark in Bezug auf die Geschlechter. Für Männer stellt der Fakt, Arbeit zu haben oder nicht, die zentrale Demarkationslinie dar und erst in einem zweiten Schritt wird auch das Wie der Arbeitsbedingungen relevant gemacht. Die Zentralität von Arbeit im Kontrast zur fortgesetzten Arbeitslosigkeit vieler anderer ehemals in der Baubranche Tätiger kommt in dem folgenden Zitat von Antonio anschaulich zum Ausdruck.

Wir könnten sagen, dass es [die Krise] vorbei ist, weil wir Arbeit haben. Aber es gibt viele unter uns, die keine [Arbeit] haben. Sie befinden sich in dem Zustand, in dem wir uns vor einigen Monaten befanden. Natürlich, wenn du Arbeit hast, bist du ruhig, weil du weißt, dass du am Ende des Monats bezahlt wirst. Aber wenn du keine [Arbeit] hast, kannst du nicht in Frieden leben. (Antonio, Grupo Boliviano 29.01.2017, S. 17)

Arbeit zu haben, wird in der Baubranche nicht als Normalität, sondern - wie in anderen Interviews wörtlich zum Ausdruck gebracht - als „Glück“ hinsichtlich der Arbeitslosigkeit vieler anderer angesehen. Wie das Zitat verdeutlicht, stellt die nach wie vor hohe Anzahl an Arbeitslosen auch für diejenigen, die Arbeit haben, eine Bedrohung dar und setzt sie - wie im Laufe der Interviews deutlich wird - unter Druck, prekäre Arbeitsbedingungen zu akzeptieren. Die interviewten Bauarbeiter klagen insbesondere darüber, dass sie nur befristete Verträge von einigen Monaten haben und nur halbtags angestellt und bezahlt werden, aber Vollzeit arbeiten müssen. Einige Befragte berichten, dass sie sich gezwungen sahen, eine Arbeit unter informellen Bedingungen anzunehmen, wodurch sie ihren Anspruch auf Sozialversicherung verloren haben. Diese Erfahrungen korrespondieren mit Analysen des 
spanischen Arbeitsmarkts, die zeigen, dass temporäre Verträge und Teilzeitarbeit zunehmend als Anpassungsmechanismus und Unternehmensstrategie zur Minimierung der Arbeitskosten eingesetzt wurden (Banyuls und Recio 2017, S. 138). Im Vergleich der Interviews sprechen Frauen von Beginn an offener über Unsicherheit und Instabilität trotz Arbeit und differenzieren stärker zwischen verschiedenen Arbeitsbedingungen. In den Erzählungen der Interviewten erscheint die Krise 2008 zudem weniger als eine Wasserscheide, sondern als ein Kontinuum der Prekarität. So betont zum Beispiel Lucia, dass ,,praktisch gesehen [...] die Hausangestellten nie ein Recht auf irgendetwas [hatten]“. Ganz ähnlich berichtet Fernanda: ,die Arbeit im Haushalt war schon immer sehr prekär und in der totalen Krise“. Gleichzeitig räumen die meisten Interviewten ein, dass sich die Arbeitsbedingungen in dem Sektor seit 2008 noch weiter verschlechtert haben. Durch Einsparungen in den privaten Haushalten wurden die Löhne über Kürzungen der Arbeitsstunden weiter gedrückt. Das entspricht Arbeitsmarktdaten, die zeigen, dass sich insbesondere in einer zweiten Phase der Krise die Bedingungen in der bezahlten Hausarbeit erheblich verschlechtert haben; so hat sich beispielsweise der Anteil der Teilzeitverträge erhöht und die Löhne sind gesunken (UGT 2016). Die Gleichzeitigkeit von Kontinuität und Vertiefung von Prekarität zeigt daher, dass die generalisierende Aussage, für Frauen gebe es immer Arbeit, die Bedingungen, unter denen diese Arbeit stattfindet, ausblendet. Die Tatsache, dass viele der interviewten Frauen trotzdem in der bezahlten Haushaltsarbeit verbleiben und kaum Männer in diesen Sektor gewechselt haben, deutet auf die tief verankerte Feminisierung dieser Arbeit. Unter Feminisierung verstehe ich ein Klassifikations- und Hierarchisierungsprinzip, durch das bestimmte Arbeiten als reproduktiv/unqualifiziert/von geringerem wirtschaftlichen Wert abgewertet werden (Peterson 2010, S. 274). Von Bedeutung sind dabei weniger inhaltliche Gesichtspunkte als vielmehr die soziale Position der Arbeiter_innen, die sie ausführen: ,jobs are regarded as unskilled because they are feminized and not feminized because they are unskilled" (Craig et al. 1982, S. 77). Wie die Interviews zeigen, stützt sich Feminisierung auf die essentialisierende Abwertung von sogenannten männlichen und der damit zusammenhängenden Aufwertung von weiblichen Eigenschaften, die mit einer „,besonderen Eignung“ für bestimmte Arbeitsbereiche assoziiert ist. Insbesondere im Kontext der ökonomischen Krise werden vergeschlechtlichte wie ethnisierte Konstruktionen stärkeren Durchhaltevermögens bestimmter Gruppen verstärkt zur Arbeitsausbeutung verwertet. Durch den Vergleich der Interviews wird deutlich, dass die Muster der geschlechtlichen Klassifizierung und Hierarchisierung von Arbeit jenen der Konstruktion eines spezifischen „migrantischen Arbeitsvermögens“ strukturell verwandt sind, wenngleich sie nicht ineinander aufgehen.

\section{Schlussfolgerungen}

In dem Beitrag ging es mir darum, einen intersektionalen Blick auf die unterschiedliche Krisenbetroffenheit entlang von Ethnizität und Geschlecht sowohl aus der Perspektive der befragten Migrant_innen als auch vor dem Hintergrund gesellschaftstheoretischer Konzepte und makrosoziologischer Befunde zur Arbeitssegmentation zu werfen. Die theoretische Herausforderung lag dabei darin, nach der Vermittlung 
beider Perspektiven zu fragen, also die Prozesse der interaktiven Herstellung von Geschlecht und Ethnizität mit der Strukturierung sozialer Verhältnisse zusammenzudenken. Als analytisch gewinnbringend erwies es sich hierbei, den Fokus auf die im empirischen Material wiederholt auftretenden Ambivalenzen, Generalisierungen und Reifizierungen zu legen. Ich möchte nun die Zusammenführung der theoretischen Überlegungen mit den Ergebnissen der Interviewanalyse noch einmal verdichten und hierfür insbesondere die eingeführte Heuristik der ,,intersektionalen Unsichtbarkeit“ (Knapp 2012) produktiv machen.

In den subjektiven Wahrnehmungen der befragten Migrant_innen changiert der Eindruck der unterschiedlichen Krisenbetroffenheit aufgrund von Ethnizität. Zum einen wird von einer größeren Betroffenheit von Migrierten von Arbeitslosigkeit ausgegangen, die sich darin begründe, dass Arbeitgeber in Krisenzeiten nicht-migrierte Arbeiter_innen bevorzugten. Diese subjektive Wahrnehmung der Interviewten wird durch Zahlen bestätigt, die zeigen, dass Migrierte insgesamt in einem höheren Ausmaß als Spanier_innen von der kriseninduzierten Arbeitslosigkeit betroffen waren. Für Teile der Migrationsforschung erneuerte sich aufgrund dieses Befundes die Validität der Konzeption von Migrierten als „,industrielle Reservearmee“. In der Tat lenkt dieses Konzept die Aufmerksamkeit darauf, wie im Zuge des ökonomischen Booms Ende der 1990er- und Anfang der 2000er-Jahre eine aktive Anwerbung von Arbeitskräften insbesondere für den Bausektor stattfand, während mit der ökonomischen Rezession eine extrem hohe Anzahl Migrierter ihre Jobs verloren, die Zuwanderung gestoppt wurde und es zur Rückkehr und Remigration vieler ehemals in diesem Sektor Beschäftigten kam. Die entgegengesetzte, von den Interviewten ebenfalls wiederholt betonte Aussage, Migrant_innen hätten immer Arbeit, weil sie im Gegensatz zu Spanier_innen ,alles machen würden“, ist hingegen mit der segmentationstheoretischen Gegenposition kongruent, die davon ausgeht, dass Migrierte vorrangig Branchen besetzten, die von Einheimischen auch in Krisenzeiten gemieden würden (Dobson et al. 2009). Diese widersprüchlichen Deutungen verweisen auf strukturelle Ungleichzeitigkeiten. So wird aus einer makrosoziologischen Perspektive deutlich, dass die unterschiedlichen Aussagen vor dem Hintergrund einer branchen- und geschlechtsspezifischen Differenzierung eingeordnet werden müssen. Maskulinisierte Sektoren wurden aufgrund ihrer hohen Volatilität insbesondere in der ersten Phase der Krise stärker von der ökonomischen Krise getroffen als feminisierte Bereiche im Dienstleistungssektor (Gil-Alonso und Vidal-Coso 2015). Diese geschlechtliche Segmentierung des Arbeitsmarkts wird auch von den Interviewten reflektiert, wenn in Bezug auf die Krisenbetroffenheit Einigkeit über eine starke Ungleichheit zwischen Männern und Frauen besteht.

Allerdings lässt - im Sinne der Heuristik der ,intersektionalen Unsichtbarkeit“ die verbreitete Generalisierung der Interviewten, für Frauen gebe es immer Arbeit, wiederum außer Acht, dass die geringere Krisenbetroffenheit feminisierter Arbeitsfelder differenziert betrachtet werden muss. Studien zu den europäischen Arbeitsmärkten haben zwar gezeigt, dass in der ersten Phase der Krise Bereiche, in denen Männer dominieren, in einem größeren Ausmaß von Arbeitslosigkeit betroffen waren (Rubery 2015). Jedoch beeinträchtigten in einer zweiten Phase die im Zuge der Austeritätspolitiken getroffenen Kürzungen der öffentlichen Ausgaben - aufgrund ihrer größeren Konzentration im öffentlichen Sektor - insbesondere Frauen 
(ebd.). Dabei muss vor allem zwischen hoch- und niedrigqualifizierten Arbeitskräften differenziert werden. Während bei niedrig Qualifizierten die Beschäftigung von Männern besonders stark von den Krisenauswirkungen betroffen ist und die Arbeitsbedingungen qua Geschlecht konvergieren, ist dies bei hoch Qualifizierten nicht der Fall (ebd.). Außerdem wird in Spanien durch einen Blick auf die unterschiedlichen Phasen der Krise deutlich, dass in einer fortgeschrittenen Phase auch migrantische Frauen zunehmend von den Auswirkungen der Krise betroffen waren (Gil-Alonso und Vidal-Coso 2015). Um die unterschiedliche Krisenbetroffenheit differenziert in den Blick zu bringen, braucht es einen intersektionalen Blick auf den Zusammenhang von Klasse, Geschlecht und Ethnizität. Das Konzept der ,intersektionalen Unsichtbarkeit“, die ,,als eine Art des Verschwundenseins der für das Konstituierte konstitutiven Verhältnisse“ (Knapp 2012, S. 259) verstanden werden kann, sensibilisiert dafür, dass jene strukturellen Zusammenhänge, die zu einer dauerhaften Abwertung von feminisierter und ethnisierter Reproduktionsarbeit führen, ausgeblendet werden. Es braucht enstprechend einen umfassenden Blick darauf, wie Krisen auf den Zusammenhang von Produktions- und Reproduktionsverhältnisse wirken (Sauer 2010, S. 39f.). Wie sich in den Interviews zeigt, deutet die Prekarisierung sowohl der bezahlten Haushaltsarbeit als auch der unbezahlten in den Familien geleisteten sozialen Reproduktion darauf hin, dass die Krisendynamiken die Auslagerung von Kosten auf den reproduktiven Bereich verstärkt haben und hierbei Migrierte besonders betroffen sind. An den Aussagen der Interviewten wird gleichzeitig sichtbar, dass (bezahlte) Haushaltsarbeit nicht erst seit der Krise prekarisiert ist, sondern diese Externalisierung für die kapitalistische Vergesellschaftung an sich konstitutiv ist.

Zur Vermittlung von subjektiven Wahrnehmungen und strukturellen Ungleichheiten kann zusammengefasst werden, dass auf der Subjektebene die widersprüchlichen Deutungen der ethnisierten Arbeitsteilung und die Generalisierung der geschlechtlichen Unterschiede auf intersektionale Unsichtbarkeiten deuten, die nur vor dem gesellschaftstheoretischen Hintergrund des Zusammenhangs von Produktion und Reproduktion dechiffriert werden können. Werden dadurch zentrale strukturelle Mechanismen gesellschaftlicher Arbeitsteilung gut auf den Punkt gebracht, fehlen jedoch Erklärungen darüber, durch welche konkreten sozialen Prozesse bestimmte Gruppen von Arbeiter_innen auf unterschiedliche Beschäftigungsverhältnisse verteilt werden. Hierfür braucht es theoretische Perspektiven auf Intersektionalität, die ihren Blick darauf richten, wie die intersektional ungleiche Arbeitsteilung in den alltäglichen Interaktionen von Arbeitnehmer_innen und Arbeitgeber_innen sozial konstruiert und legitimiert wird. Dies geschieht durch Prozesse der Ethnisierung und Feminisierung, die - wie in den Interviews deutlich wird - vor dem subjekttheoretischen Horizont über die positive Zuschreibung essentialisierender Eigenschaften der Selbst- wie Fremdaufwertung dienen können, gesellschaftlich aber zur Produktion und Verfestigung ungleicher Arbeitsteilung beitragen. Um zu einer differenzierten Analyse von Mechanismen der Arbeitsausbeutung zu gelangen, bedarf eine intersektionale Perspektivierung gesellschaftlicher Arbeitsteilung daher eines Ansatzes, der beides berücksichtigt und verbindet: die strukturelle Ebene der Ungleichheitsverhältnisse sowie die in den alltäglichen Interaktionen konstruierten und sich institutionell verfestigenden Differenzen. Denn Ethnizität und Geschlecht sind zentrale Prinzipien gesellschaftlicher Strukturierung, die als „Platzanweiser“ die individuellen Akteure 
gesellschaftlich positionieren. Ihre konkrete Wirksamkeit und die maßgeblichen Prozesse, durch die über sie Arbeit angeeignet, ausgebeutet und verteilt wird, können jedoch nur empirisch bestimmt werden.

Funding Open access funding provided by University of Vienna.

Open Access Dieser Artikel wird unter der Creative Commons Namensnennung 4.0 International Lizenz veröffentlicht, welche die Nutzung, Vervielfältigung, Bearbeitung, Verbreitung und Wiedergabe in jeglichem Medium und Format erlaubt, sofern Sie den/die ursprünglichen Autor(en) und die Quelle ordnungsgemäß nennen, einen Link zur Creative Commons Lizenz beifügen und angeben, ob Änderungen vorgenommen wurden.

Die in diesem Artikel enthaltenen Bilder und sonstiges Drittmaterial unterliegen ebenfalls der genannten Creative Commons Lizenz, sofern sich aus der Abbildungslegende nichts anderes ergibt. Sofern das betreffende Material nicht unter der genannten Creative Commons Lizenz steht und die betreffende Handlung nicht nach gesetzlichen Vorschriften erlaubt ist, ist für die oben aufgeführten Weiterverwendungen des Materials die Einwilligung des jeweiligen Rechteinhabers einzuholen.

Weitere Details zur Lizenz entnehmen Sie bitte der Lizenzinformation auf http://creativecommons.org/ licenses/by/4.0/deed.de.

\section{Literatur}

Anderson, Bridget. 2019. New directions in migration studies: towards methodological de-nationalism. Comparative Migration Studies 7:1-13.

Anthias, Floya. 2012. Hierarchies of social location, class and intersectionality: towards a translocational frame. International Sociology 28:121-138.

Araujo, Sandra, und Claudia Pedone. 2013. Migration policies and family life across the Atlantic: Latin American migrant families in Spain. In Migration and family: theory, research and fields of action, Hrsg. Thomas Geisen, Tobias Studer, und Erol Yildiz, 341-355. Wiesbaden: VS.

Araujo, Sandra Gil, und Tania González-Fernández. 2014. International migration, public policies and domestic work: Latin American migrant women in the Spanish domestic work sector. Women's Studies International Forum 46:13-23.

Bakker, Isabella, und Gill Stephen. 2003. Global political economy and social reproduction. In Power, production and social reproduction, Hrsg. Isabella Bakker, Stephen Gill, 3-16. New York: Springer.

Banyuls, Josep, und Albert Recio. 2017. Labour segmentation and precariousness in Spain: theories and evidence. In Making work more equal. A new labour market segmentation approach for analysing inequalities, Hrsg. Damian Grimshaw, Colette Fagan, und Gail Hebson, 129-149. Manchester: Manchester University Press.

Baumer, Andreas. 2017. Irreguläre Migration und staatliche Politik in Spanien und Europa. Wiesbaden: Springer VS.

Becker-Schmidt, Regina. 2004. Doppelte Vergesellschaftung von Frauen: Divergenzen und Brückenschläge zwischen Privat- und Erwerbsleben. In Handbuch Frauen- und Geschlechterforschung, Hrsg. Ruth Becker, Beate Kortendiek, 62-71. Wiesbaden: VS.

Becker-Schmidt, Regina. 2009. Vortragsmanuskript Kassel. Zitiert in: Knapp, Gudrun-Axeli. 2013. „Intersectional Invisibility“: Anknüpfungen und Rückfragen an ein Konzept der Intersektionalitätsforschung. In Fokus Intersektionalität, Hrsg. Helma Lutz, Maria Teresa Herrera Vivar, und Linda Supik, 243-264. Wiesbaden: VS.

Beets, Gijs, und Frans Willekens. 2009. The global economic crisis and international migration: an uncertain outlook. Vienna Yearbook of Population Research 7:19-37.

Bohnsack, Ralf. 2010. Rekonstruktive Sozialforschung. Einführung in Qualitative Methoden. Stuttgart: UTB.

Buckel, Sonja. 2012. „Managing Migration“ - Eine intersektionale Kapitalismusanalyse am Beispiel der Europäischen Migrationspolitik. Berliner Journal für Soziologie 22:79-100.

Cachón, Lorenzo, und María Aysa-Lastra. 2015. Native and Latino employment during the great recession in the US and Spain. In Immigrant Vulnerability and Resilience: Comparative Perspectives on Latin 
American Immigrants during the Great Recession, Hrsg. María Aysa-Lastra, und Lorenzo Cachón, 25-46. Cham: Springer.

Castles, Stephen. 2011. Migration, crisis, and the global labour market. Globalizations 8:311-324.

Collins, Patricia Hill, und Sirma Bilge. 2016. Intersectionality. Cambridge: Polity.

Craig, Christine, Jill Rubery, Roger Tarling, und Frank Wilkinson. 1982. Labour market structure, industrial organisation and low pay. Cambridge: Cambridge University Press.

Degele, Nina, und Gabriele Winker. 2011. Intersektionalität als Beitrag zu einer gesellschaftstheoretisch informierten Ungleichheitsforschung. Berliner Journal für Soziologie 21:69-90.

De Genova, Nicholas. 2013. Spectacles of migrant 'illegality': the scene of exclusion, the obscene of inclusion. Ethnic and Racial Studies 36:1180-1198.

Demirovic, Alex, und Andrea Maihofer. 2013. Vielfachkrise und die Krise der Geschlechterverhältnisse. In Krise, Kritik, Allianzen. Arbeits- und geschlechtersoziologische Perspektiven, Hrsg. Hildegard Maria Nickel, und Andreas Heilmann, 30-48. Weinheim: Beltz Juventa.

Dobson, Janet, Alan Latham, und John Salt. 2009. On the move? Labour migration in times of recession. Policy network paper, 1-23.

Dörre, Klaus, Michael Behr, Dennis Eversberg, und Karen Schierhorn. 2009. Krise ohne Krisenbewusstsein? PROKLA. Zeitschrift für Kritische Sozialwissenschaft 39:559-576.

Díaz Gorfinkiel, Magdalena. 2016. La reconfiguración del empleo doméstico en España: impacto de los cambios legislativos en el funcionamiento del sector. Revista Española de Investigaciones Sociológicas 155:97-112.

Farris, Sara. 2012. Femonationalism and the 'regular' army of labor called migrant women. History of the Present 2:184-199.

Gil-Alonso, Fernando, und Elena Vidal-Coso. 2015. Inmigrantes extranjeros en el mercado de trabajo español: ¿Más resilientes o más vulnerables al impacto de la crisis? Migraciones 37:97-123.

Gutiérrez Rodríguez, Encarnación. 2005. Das postkoloniale Europa dekonstruieren. Zur Prekarisierung, Migration und Arbeit. Widerspruch 48:71-84.

Hudson, Maria, Gina Netto, Mike Noon, Filip Sosenko, Philomena de Lima, und Nicolina KamenouAigbekaen. 2017. Ethnicity and low wage traps: favouritism, homosocial reproduction and economic marginalization. Work, Employment and Society 31:992-1009.

Jacobo, Muñoz. 2012. Evolución del empleo y del paro de las mujeres inmigrantes en el mercado de trabajo español. Cuadernos de Relaciones Laborales 30:115-137.

Jorba, Marta, und Maria Rodó-Zárate. 2019. Beyond mutual constitution: the properties framework for intersectionality studies. Signs: Journal of Women in Culture and Society 45:175-200.

Kamberelis, George, und Greg Dimitriadis. 2013. Focus groups: from structured interviews to collective conversations. London, New York: Routledge.

Klinger, Cornelia. 2003. Ungleichheit in den Verhältnissen von Klasse, Rasse und Geschlecht. In Achsen der Differenz: Gesellschaftstheorie und feministische Kritik II, Hrsg. Gudrun-Axeli Knapp, und Angelika Wetterer, 14-48. Münster: Westfälisches Dampfboot.

Knapp, Gudrun-Axeli. 2012. Im Widerstreit. Feministische Theorie in Bewegung. Wiesbaden: VS.

Knapp, Gudrun-Axeli. 2013. „Intersectional Invisibility“: Anknüpfungen und Rückfragen an ein Konzept der Intersektionalitätsforschung. In Fokus Intersektionalität, Hrsg. Helma Lutz, Maria Teresa Herrera Vivar Helma, und Linda Supik, 243-264. Wiesbaden: VS.

Kofman, Eleonore. 2013. Gendered labour migrations in Europe and emblematic migratory figures. Journal of Ethnic and Migration Studies 39:579-600.

McBride, Anne, Gail Hebson, und Jane Holgate. 2015. Intersectionality: are we taking enough notice in the field of work and employment relations? Work, employment and society 29:331-341.

McKinzie, Ashleigh, und Patricia Richards. 2019. An argument for context-driven intersectionality. Sociology Compass 13:1-14.

Meardi, Guglielmo, Antonio Martin, und Mariona Lozano Riera. 2012. Constructing uncertainty: Unions and migrant labour in construction in Spain and the UK. Journal of Industrial Relations 54:5-21.

Mezzadra, Sandro. 2011. The gaze of autonomy: capitalism, migration and social struggles. In The contested politics of mobility: Borderzones and irregularity, Hrsg. Vicki Squire, 121-142. London: Routledge.

Neuhauser, Johanna. 2018. 'The crisis is over? Maybe for the rich, but not for us!': latin American migrants' responses to the (post-)crisis discourse in Spain. Sociology 52:1-16.

Orrenius, Pia M., und Madeline Zavodny. 2009. Tied to the business cycle: how immigrants fare in good and bad economic times. Washington, DC: Migration Policy Institute.

Oso, Laura, und Catarino Cristina. 2013. From sex to gender: The feminisation of migration and labour market insertion in Spain and Portugal. Journal of Ethnic and Migration Studies 39:625-647. 
Oxfam Intermón. 2017. Una Economía para el 99\%. España, un Crecimiento Económico que Deja Fuera a las Personas Vulnerables. https://www.oxfam.org/es/informes/una-economia-para-el-99. Zugegriffen: 17. Nov. 2018.

Peck, Jamie. 1996. Work-place. The social regulation of labor markets. New York: Guilford.

Peterson, V. Spike. 2010. Global householding amid global crises. Politics \& Gender 6(2):271-281.

Pflücke, Virginia Kimey. 2018. Wenn Hausarbeit bezahlt wird: Der Wandel der Arbeitsbeziehung im Privathaushalt in Spanien und Uruguay (= Labour Studies, Bd. 20). Frankfurt am Main: Campus.

Pollert, Anna. 1996. Gender and class revisited; or, the poverty of 'patriarchy'. Sociology 30:639-659.

Quijano, Aníbal. 2016. Kolonialität der Macht, Eurozentrismus und Lateinamerika. Wien: Turia und Kant.

Reher, David, Miguel Requena, und Alberto Sanz. 2011. España en la encrucijada? Consideraciones sobre el cambio de ciclo migratorio. Revista Internacional de Sociología 1:9-44.

Román, Salvador Manzanera. 2018. Las políticas del mercado de trabajo como factor de intensificación de precariedad laboral y exclusión social. Prisma Social: Revista De Investigación Social 23:331-352.

Rosnick, David, und Mark Weisbrot. 2015. Has Austerity Worked in Spain? CEPRReports and Issue Briefs 2015-27, Center for Economic and Policy Research (CEPR). http://gesd.free.fr/ceprspain.pdf. Zugegriffen: 12. Dez. 2016.

Ross, Bettina. 2008. Intersektionale Perspektiven auf Internationale Arbeitsteilung. Femina Politica $17: 29-40$.

Rubery, Jill. 2015. Austerity and the future for gender equality in Europe. ILR Review 6:715-741.

Sauer, Birgit. 2010. Das Geschlecht der Finanz- und Wirtschaftskrise. Kurswechsel 1:38-46.

Serrano, Carlos García. 2012. Del pasmo al marasmo: El sector de la construcción y su relación con la crisis del empleo. Estudios de economía aplicada 30:163-182.

Tapia, Maite, und Gabriella Alberti. 2019. Unpacking the category of migrant workers in trade union research: a multi-level approach to migrant intersectionalities. Work, Employment and Society 33:314-325.

Teubner, Ulrike. 2010. Beruf. In Handbuch Frauen- und Geschlechterforschung, Hrsg. Ruth Becker, und Beate Kortendiek, 499-506. Wiesbaden: VS.

Torres, Francisco, und M. Elena Gadea. 2015. Un proceso de inserción desestabilizado por la crisis: El nuevo ciclo migratorio y las estrategias de los inmigrantes. In Crisis, inmigración y sociedad, Hrsg. Francisco Torres, und M. Elena Gadea, 9-35. Madrid: Talasa Ediciones.

UGT Unión General de Trabajadores. 2016. Trabajo decente para las trabajadoras y trabajadores domésticos. http://www.ugt.es/Publicaciones/Informe_trabajo_decente_para_trabajadoras_trabajadores_ domesticos_UGT.pdf. Zugegriffen: 4. Febr. 2017.

Waldinger, Roger, und Michael Lichter. 2003. How the other half works: Immigration and the social organization of labor. Berkeley: University of California Press.

Wetterer, Angelika. 2002. Arbeitsteilung und Geschlechterkonstruktionen: Gender at Work in theoretischer und historischer Perspektive. Konstanz: UVK.

Wichterich, Christa. 2010. Geschlechteranalysen und -diskurse in der Krise. Peripherie 118/119:164-187.

Johanna Neuhauser Dr. rer. pol., arbeitet als Universitätsassistentin (Post-Doc) am Institut für Soziologie der Universität Wien. Davor war sie am Institut für Migrationsforschung und Interkulturelle Studien (IMIS) an der Universität Osnabrück tätig und hat an der Unversität Kassel in Soziologie promoviert. Forschungsschwerpunkte: Arbeitsmigration und migrantische Arbeit, Geschlecht und Intersektionalität, Globale Ungleichheit. 